\title{
THE GENERIC INITIAL IDEALS OF POWERS OF A 2-COMPLETE INTERSECTION
}

\author{
SARAH MAYES
}

\begin{abstract}
We compute the reverse lexicographic generic initial ideals of the powers of a 2-complete intersection ideal I. In particular, we present six algorithms to compute these generic initial ideals; the power and the relative degrees of the minimal generators of $I$ determine the algorithm to be used.
\end{abstract}

1. Introduction. Consider the collection of ideals $\left\{\operatorname{gin}\left(I^{n}\right)\right\}$ obtained by taking the generic initial ideals of powers of a fixed ideal $I$ in a polynomial ring. Our study of such families of monomial ideals was initially motivated by the desire to understand their asymptotic behavior (see [13]). It soon became clear, however, that the individual ideals within such families are interesting in their own right. In this paper, we compute the generators of the ideals gin $\left(I^{n}\right)$ with respect to the reverse lexicographic order where $I$ is a 2-complete intersection.

Computing generic initial ideals is generally challenging because they are defined by an existence theorem rather than an explicit construction (see Galligo's theorem, Theorem 2.1). As a result, there are few classes of ideals for which generic initial ideals have been explicitly computed (see [10] for a survey, or $[\mathbf{6}, \mathbf{1}, \mathbf{5}, \mathbf{7}]$ for more recent results).

The 2-complete intersections are amongst the ideals whose reverse lexicographic generic initial ideals are completely understood. In particular, if $I \subset K\left[x_{1}, \ldots, x_{m}\right]$ is generated by a regular sequence of homogeneous polynomials of degrees $\alpha$ and $\beta$, with $\alpha \leq \beta$, then

$$
\operatorname{gin}(I)=\left(x_{1}^{\alpha}, x_{1}^{\alpha-1} x_{2}^{\lambda_{0}-2(\alpha-1)}, x_{1}^{\alpha-2} x_{2}^{\lambda_{0}-2(\alpha-2)}, \ldots, x_{1} x_{2}^{\lambda_{0}-2}, x_{2}^{\lambda_{0}}\right)
$$

where $\lambda_{0}=\beta+\alpha-1$ (see [10, Section 4]). The generic initial ideals for larger complete intersections, however, have proved difficult to compute. For example, Cimpoeaş [6] has exhibited the minimal

2010 AMS Mathematics subject classification. Primary 13P10, Secondary $13 \mathrm{C} 40$.

Received by the editors on May 1, 2013, and in revised form on March 4, 2014. DOI: 10.1216/JCA-2015-7-1-55 Copyright (c)2015 Rocky Mountain Mathematics Consortium 
generators for the generic initial ideals of strongly Lefschetz 3-complete intersections; the structure of such generic initial ideals is difficult to describe and depends on the relative degrees of the generators of the complete intersection.

In this paper, we explicitly compute the generators of the reverse lexicographic generic initial ideals of powers of 2-complete intersections. In particular, we prove the following result.

Theorem 1.1. Fix positive integers $\alpha, \beta$ and $n$ such that $\beta \geq \alpha$ and $n \geq 2$. Compute the sequence of invariants $\left\{\lambda_{i}\right\}$ using:

- Algorithm 1 if $\beta \geq 2 \alpha-1$ :

- Algorithm 2 if $2 \alpha-1>\beta \geq(3 / 2) \alpha$ :

- Algorithm 3 if $(3 / 2) \alpha>\beta>\alpha,(\beta-\alpha) \mid \alpha$, and $n \geq$ $\alpha /(\beta-\alpha)+1$ :

- Algorithm 4 if $(3 / 2) \alpha>\beta>\alpha,(\beta-\alpha) \nmid \alpha$, and $n \geq$ $\lceil\alpha /(\beta-\alpha)\rceil+1:$

- Algorithm 5 if $(3 / 2) \alpha>\beta>\alpha$ and $2 \leq n<\lceil\alpha /(\beta-\alpha)\rceil+1$ : and

- Algorithm 6 if $\alpha=\beta$.

If I is a type $(\alpha, \beta)$ complete intersection in $R$ then, setting $x=x_{1}$ and $y=x_{2}$, the reverse lexicographic generic initial ideal of $I^{n}$ is

$$
\operatorname{gin}\left(I^{n}\right)=\left(x^{k}, x^{k-1} y^{\lambda_{k-1}}, \ldots, x y^{\lambda_{1}}, y^{\lambda_{0}}\right)
$$

where $k=n \alpha$.

The algorithms referred to in this theorem are stated in Section 4 . Although the algorithm that will be used in a particular case depends on $n$ and on the relative sizes of $\alpha$ and $\beta$, all of the algorithms share common features. For example, they each compute the invariants $\lambda_{i}$ one-by-one, starting with $\lambda_{0}=n \beta+\alpha-1$ and using the gaps $g_{i}:=\lambda_{i-1}-\lambda_{i}$ between the $\lambda_{i}$ to compute each successive invariant. The patterns within the invariants of the ideals gin $\left(I^{n}\right)$ are best seen by looking at the associated gap sequences $\left\{g_{i}\right\}$, which consist entirely of the numbers 1,2 , and $\beta-2 \alpha+2$.

This theorem adds powers of 2-complete intersections to the classes of ideals whose generic initial ideals can be explicitly computed. The complexity of this result even in this small case gives further evidence 
that finding generators of the generic initial ideals of powers of larger complete intersections is extremely difficult, thus providing motivation to instead study the asymptotic behavior of generic initial systems $\left\{\operatorname{gin}\left(I^{n}\right)\right\}_{n}$. Indeed, the asymptotic behaviour of such systems can be remarkably simple (see subsection 4.3).

The following section contains general background information on generic initial ideals, the Hilbert function and notation used in this paper. Subsection 3.1 establishes the form of the generic initial ideal of a power of a 2-complete intersection and subsection 3.2 lays out important facts about the Hilbert functions of such ideals that will be used in the proof of the main theorem. The algorithms referred to in Theorem 1.1 are stated and analyzed in Section 4. Finally, Section 5 contains an overview of the proof of Theorem 1.1 and details of the proof in a representative case.

2. Preliminaries. In this section, we will introduce some notation, definitions and preliminary results related to generic initial ideals. Throughout, $R=K\left[x_{1}, \ldots, x_{m}\right]$ is a polynomial ring over a field $K$ of characteristic 0 with the standard grading and some fixed term order $>$ with $x_{1}>x_{2}>\cdots>x_{m}$.

2.1. Generic initial ideals. An element $g=\left(g_{i j}\right) \in \mathrm{GL}_{m}(K)$ acts on $R$ and sends any homogeneous element $f\left(x_{1}, \ldots, x_{m}\right)$ to the homogeneous element $f\left(g\left(x_{1}\right), \ldots, g\left(x_{m}\right)\right)$ where $g\left(x_{i}\right)=\sum_{j=1}^{m} g_{i j} x_{j}$. If $g(I)=I$ for every upper triangular matrix $g$, then we say that $I$ is Borel-fixed. Borel-fixed ideals are strongly stable when $K$ is of characteristic 0 ; that is, for every monomial $f$ in the ideal such that $x_{i}$ divides $f$, the monomials $x_{j} f / x_{i}$ for all $j<i$ are also in the ideal.

To any homogeneous ideal $I$ of $R$ we can associate a Borel-fixed monomial ideal $\operatorname{gin}_{>}(I)$ which can be thought of as a coordinateindependent version of the initial ideal. Its existence is guaranteed by the following result known as Galligo's theorem (also see [10, Theorem $1.27])$.

Theorem $2.1([4,9])$. For any multiplicative monomial order $>$ on $R$ and any homogeneous ideal $I \subset R$, there exists a Zariski open subset $U \subset \mathrm{GL}_{m}$ such that $\mathrm{In}_{>}(g(I))$ is constant and Borel-fixed for all $g \in U$. 
Definition 2.2. The generic initial ideal of $I$, denoted $\operatorname{gin}_{>}(I)$, is defined to be $\operatorname{In}_{>}(g(I))$ where $g \in U$ is as in Galligo's theorem.

The reverse lexicographic order $>$ is a total ordering on the monomials of $R$ defined by:

(1) if $|I|=|J|$, then $x^{I}>x^{J}$ if there is a $k$ such that $i_{\ell}=j_{\ell}$ for all $\ell>k$ and $i_{\ell}<j_{\ell} ;$ and

(2) if $|I|>|J|$, then $x^{I}>x^{J}$.

For example, $x_{1}^{2} x_{3}<x_{1} x_{2}^{2}$. From this point on, $\operatorname{gin}(I)=\operatorname{gin}_{>}(I)$ will denote the generic initial ideal with respect to the reverse lexicographic order.

2.2. The Hilbert function and notation. Recall that the Hilbert function $H_{I}(t)$ of a homogeneous ideal $I$ is defined by $H_{I}(t)=\operatorname{dim}_{K}\left(I_{t}\right)$ where $I_{t}$ denotes the $t$ th graded piece of $I$. The following theorem records two of the properties shared by gin $(I)$ and $I$. The first statement is a consequence of the fact that Hilbert functions are invariant under making changes of coordinates and taking initial ideals. The second statement is a result of Bayer and Stillman [3]; for a simple proof see [2, Corollary 2.8].

Theorem 2.3. For any homogeneous ideal I in $R$ :

(1) the Hilbert functions of $I$ and gin $(I)$ are equal; and

(2) under the reverse lexicographic order, $\operatorname{depth}(R / I)=\operatorname{depth}(R / \operatorname{gin}(I))$.

Throughout this paper, $\left(\begin{array}{l}s \\ t\end{array}\right)=0$ whenever $s \leq 0$ or $t>s$ so that $\left(\begin{array}{l}s \\ t\end{array}\right)$ is always nonnegative. Under this assumption, the summation and recursive formulas for binomial coefficients hold. Finally, since most of our work will only involve the first two variables $x_{1}$ and $x_{2}$ of $K\left[x_{1}, \ldots, x_{m}\right]$, we will set $x_{1}=x$ and $x_{2}=y$.

3. Structure of ideals in the generic initial system. A homogeneous ideal $I=\left(f_{\alpha}, f_{\beta}\right)$ is a complete intersection of type $(\alpha, \beta)$ if $f_{\alpha}, f_{\beta}$ is a regular sequence on $R, \operatorname{deg}\left(f_{\alpha}\right)=\alpha$, and $\operatorname{deg}\left(f_{\beta}\right)=\beta$. Since $f_{\beta}, f_{\alpha}$ is also a regular sequence, we may assume that $\alpha \leq \beta$. Throughout this section we assume that $I$ is such a complete intersection. 
3.1. Structure of gin $\left(I^{n}\right)$. The goal of this subsection is to describe the general structure of the reverse lexicographic generic initial ideals gin $\left(I^{n}\right)$ for a complete intersection $I$ of type $(\alpha, \beta)$. In particular, we will prove the following theorem.

Theorem 3.1. Let $I$ be a complete intersection of type $(\alpha, \beta)$ in $R=K\left[x_{1}, \ldots, x_{m}\right]$ generated by the homogeneous polynomials $f_{\alpha}$ and $f_{\beta}$, and suppose that $A_{n}$ is the set of minimal monomial generators of $\operatorname{gin}\left(I^{n}\right)$. Then, setting $x=x_{1}$ and $y=x_{2}$,

$$
A_{n}=\left\{x^{k}, x^{k-1} y^{\lambda_{k-1}}, x^{k-2} y^{\lambda_{k-2}}, \ldots, x y^{\lambda_{1}}, y^{\lambda_{0}}\right\}
$$

where:

(i) $\lambda_{0}>\lambda_{1}>\cdots>\lambda_{k-2}>\lambda_{k-1}$;

(ii) $k=n \alpha$;

(iii) $\lambda_{0}=n \beta+\alpha-1$; and

(iv) $\lambda_{k-1}=\beta-\alpha+1$.

We will refer to the $\lambda_{i}$ as the invariants of $\operatorname{gin}\left(I^{n}\right)$. This theorem will be proven in several parts. First, no matter how many variables the ambient ring $R$ has, the minimal generators of these generic initial ideals will only involve the variables $x_{1}$ and $x_{2}$.

Lemma 3.2. Let $I$ be a type $(\alpha, \beta)$ complete intersection in $R$, and let $A_{n}$ denote the set of minimal monomial generators of $\operatorname{gin}\left(I^{n}\right)$. Then the elements of $A_{n}$ are contained in $K\left[x_{1}, x_{2}\right]$. Furthermore, $A_{n}$ contains a power of $x_{2}$, say $x_{2}^{\lambda_{0}}$, and no element of $A_{n}$ is of degree greater than $\lambda_{0}$.

This lemma is a consequence of the following result of Herzog and Srinivasan (see [12, Lemma 3.1]) which relates the depth and dimension of a Borel-fixed monomial ideal to the variables appearing in its minimal generating set.

Proposition 3.3 ([12]). Let $J$ be a Borel-fixed monomial ideal in $R$, and define

$$
D(J):=\max \left\{t \mid x_{t}^{j} \in J \text { for some positive integer } j\right\}
$$


and

$M(J):=\max \left\{t \mid x_{t}\right.$ appears in some minimal generator of $\left.J\right\}$. Then $\operatorname{dim}(R / J)=m-D(J)$ and $\operatorname{depth}(R / J)=m-M(J)$.

Note that, when $I$ is a complete intersection of type $(\alpha, \beta)$ in $R$, $\operatorname{dim}\left(R / I^{n}\right)=\operatorname{depth}\left(R / I^{n}\right)=m-2$ for all $n \geq 1$. It then follows by Theorem 2.3 that the depth and dimension of $R / \operatorname{gin}\left(I^{n}\right)$ are equal to $m-2$ as well.

Proof of Lemma 3.2. By Proposition 3.3,

$$
\begin{aligned}
D\left(\operatorname{gin}\left(I^{n}\right)\right) & =m-\operatorname{dim}\left(R / \operatorname{gin}\left(I^{n}\right)\right)=2 \\
& =m-\operatorname{depth}\left(R / \operatorname{gin}\left(I^{n}\right)\right)=M\left(\operatorname{gin}\left(I^{n}\right)\right) .
\end{aligned}
$$

This means that the minimal monomial generating set $A_{n}$ of $\operatorname{gin}\left(I^{n}\right)$ is contained in $S=K\left[x_{1}, x_{2}\right]$ and that $A_{n}$ contains a power of $x_{2}$, say $x_{2}^{\lambda_{0}}$. The final statement follows from the fact that gin $\left(I^{n}\right)$ is strongly stable.

Proof of Theorem 3.1 (i). This is a direct consequence of Lemma 3.2 and the fact that generic initial ideals are strongly stable.

Proof of Theorem 3.1 (ii). Note that the homogeneous polynomial $f_{\alpha}^{n}$ is an element of $I^{n}$ of the smallest degree possible. Under a general change of coordinates $g$, the smallest degree element of $g\left(I^{n}\right)$ is also of degree $n \alpha$. Thus, the smallest degree element of in $\left(g\left(I^{n}\right)\right)=\operatorname{gin}\left(I^{n}\right)$ has degree $n \alpha$ and, since gin $\left(I^{n}\right)$ is strongly stable, this is equal to the power of $x$ in $A_{n}$.

To determine the values of $\lambda_{0}$ and $\lambda_{k-1}$, we will compare the Betti numbers of $I^{n}$ and gin $\left(I^{n}\right)$ using the 'cancellation principle.' Let

$$
0 \longrightarrow F_{m} \longrightarrow \cdots \longrightarrow F_{1} \longrightarrow F_{0} \longrightarrow J \longrightarrow 0
$$

be the unique minimal free graded resolution of a homogeneous ideal $J$. The graded Betti numbers of $J, \beta_{i, j}(J)$, are defined by $F_{i}=$ $\bigoplus_{j} R(-j)^{\beta_{i, j}(J)}$. A consecutive cancellation takes a sequence $\left\{\beta_{i, j}\right\}$ to a new sequence by replacing $\beta_{i, j}$ by $\beta_{i, j}-1$ and $\beta_{i+1, j}$ by $\beta_{i+1, j}-1$. The cancellation principle says that the graded Betti numbers $\beta_{i, j}\left(I^{n}\right)$ 
of $I^{n}$ can be obtained from the graded Betti numbers $\beta_{i, j}\left(\operatorname{gin}\left(I^{n}\right)\right)$ of gin $\left(I^{n}\right)$ by making a series of consecutive cancellations (see [10, Corollary 1.21]).

In order to apply the cancellation principle to find $\lambda_{k-1}$ and $\lambda_{0}$, we need to know the Betti numbers of $I^{n}$ and an ideal having the same form as gin $\left(I^{n}\right)$; this information is recorded in the following two propositions.

Proposition 3.4 ([11]). Suppose that I is a complete intersection of type $(\alpha, \beta)$. Then the minimal free resolution of $I^{n}$ is of the form

$$
0 \longrightarrow \mathcal{H}_{1} \longrightarrow \mathcal{H}_{0} \longrightarrow I^{n} \longrightarrow 0
$$

where

$$
\mathcal{H}_{1}=\bigoplus_{p=1}^{n} R(-\alpha p-\beta(n+1-p))
$$

and

$$
\begin{aligned}
\mathcal{H}_{0} & =\bigoplus_{p=0}^{n} R(-\alpha p-\beta(n-p)) \\
& =R(-\alpha n) \oplus \bigoplus_{p=0}^{n-1} R(-\alpha p-\beta(n-p)) .
\end{aligned}
$$

Proposition 3.5 (cf. [8]). The minimal free resolution of $J=$ $\left(x^{k}, x^{k-1} y^{\lambda_{k-1}}, \ldots, x y^{\lambda_{1}}, y^{\lambda_{0}}\right)$ where $\lambda_{0}>\lambda_{1}>\cdots \lambda_{k-1}$ is of the form

$$
0 \longrightarrow \mathcal{G}_{1} \longrightarrow \mathcal{G}_{0} \longrightarrow J \longrightarrow 0,
$$

where

$$
\mathcal{G}_{1}=\bigoplus_{i=0}^{k-1} R\left(-\lambda_{i}-i-1\right)
$$

and

$$
\mathcal{G}_{0}=\left(\bigoplus_{i=0}^{k-1} R\left(-\lambda_{i}-i\right)\right) \oplus R(-k)
$$


Proof of Theorem 3.1 (iii). Since the invariants $\lambda_{i}$ are strictly decreasing, $\lambda_{0}+1>\lambda_{i}+i \geq k$ for $i=0, \ldots, k-1$. Thus, if $\left\{\beta_{i, j}\right\}$ is the set of graded Betti numbers of $\operatorname{gin}\left(I^{n}\right), \beta_{1, \lambda_{0}+1} \geq 1$ and $\beta_{0, \lambda_{0}+1}=0$ by Proposition 3.5. Therefore, no consecutive cancellation can replace $\beta_{1, \lambda_{0}+1}$, and, after any series of consecutive cancellations,

$$
\max \left\{t \mid \beta_{1, t} \geq 1\right\}=\lambda_{0}+1 \text {. }
$$

By Proposition 3.4, $\alpha+n \beta$ is the largest shift in $\mathcal{H}_{1}$. Thus, by the cancellation principle, $\lambda_{0}+1=\alpha+n \beta$, or $\lambda_{0}=\alpha+n \beta-1$.

Proof of Theorem 3.1 (iv). Since the invariants $\lambda_{i}$ are strictly decreasing and $\lambda_{k-1} \geq 1, k \leq \lambda_{k-1}+(k-1)<\lambda_{i}+i+1$ for all $i=0, \ldots, k-1$. Thus, if $\left\{\beta_{i, j}\right\}$ is the set of graded Betti numbers of gin $\left(I^{n}\right), \beta_{0, k} \geq 1, \beta_{0, \lambda_{k-1}+k-1} \geq 1, \beta_{1, k}=0$ and $\beta_{1, \lambda_{k-1}+k-1}=0$ by Proposition 3.5. Therefore, no consecutive cancellation can replace $\beta_{0, k}$ or $\beta_{0, \lambda_{k-1}+k-1}$ and, for every $t$ such that $t<k$ or $k<t<\lambda_{k-1}+k-1$, $\beta_{0, t}=0$ (note that it is possible to have $k=\lambda_{k-1}+k-1$ ).

By Proposition 3.4, the two smallest shifts in $\mathcal{H}_{0}$ are $n \alpha$ and $\alpha(n-1)+\beta$. Thus, by the cancellation principle, $k=n \alpha$ (as we have seen in the proof of part (ii)) and $\lambda_{k-1}+k-1=\lambda_{n \alpha-1}+n \alpha-1=$ $\alpha(n-1)+\beta$, or

$$
\lambda_{n \alpha-1}=\beta-\alpha+1 \text {. }
$$

Note that we can write $\lambda_{0}$ and $\lambda_{k-1}$ in terms of $l:=\beta-\alpha$ and $\alpha$ as follows:

$$
\begin{gathered}
\lambda_{0}=n(\alpha+l)+\alpha-1=(n+1) \alpha+n l-1 \\
\lambda_{k-1}=\lambda_{n \alpha-1}=\beta-\alpha+1=l+1 .
\end{gathered}
$$

3.2. The Hilbert function of $\operatorname{gin}\left(I^{n}\right)$. The following result tells us that the invariants of gin $\left(I^{n}\right)$ are completely determined by $H_{\operatorname{gin}\left(I^{n}\right)}(t)$; this observation will be the key to computing these invariants.

Lemma 3.6. Suppose that we have an ideal $J$ of the form

$$
J=\left(x^{k}, x^{k-1} y^{\mu_{k-1}}, \ldots, x y^{\mu_{1}}, y^{\mu_{0}}\right)
$$

where the $\mu_{i} s$ are strictly decreasing. If $H_{J}(t)=H_{I^{n}}(t)$ for a type $(\alpha, \beta)$ complete intersection ideal $I$, then $\operatorname{gin}\left(I^{n}\right)=J$. 
This lemma is an immediate consequence of the following well-known result (see, for example, [10, Lemma 4.2]).

Lemma 3.7. An ideal of the form $J=\left(x^{k}, x^{k-1} y^{\lambda_{k-1}}, \ldots, x y^{\lambda_{1}}, y^{\lambda_{0}}\right)$ where $\lambda_{0}>\lambda_{1}>\cdots>\lambda_{k-1}$ is uniquely determined by its Hilbert function.

Proof of Lemma 3.6. By Theorem 2.3,

$$
H_{\operatorname{gin}\left(I^{n}\right)}(t)=H_{I^{n}}(t)=H_{J}(t) .
$$

Since, $J$ and gin $\left(I^{n}\right)$ are both of the form considered in Lemma 3.7, they are uniquely determined by their Hilbert functions and $J=$ $\operatorname{gin}\left(I^{n}\right)$.

To prove that the numbers $\left\{\lambda_{i}\right\}$ produced by the algorithms presented in Section 4 are indeed the invariants of gin $\left(I^{n}\right)$, we will compute the Hilbert function of the ideal

$$
J=\left(x^{k}, x^{k-1} y^{\lambda_{k-1}}, \ldots, x y^{\lambda_{1}}, y^{\lambda_{0}}\right) .
$$

By Lemma 3.6, it is then sufficient to show that $H_{J}(t)$ is equal to $H_{I^{n}}(t)$. We will now record expressions for the Hilbert functions of $I^{n}$ and $J$ that will be used to carry out this procedure; the following two propositions follow from Propositions 3.4 and 3.5, respectively.

Proposition 3.8. If I is the ideal of a type $(\alpha, \beta)$ complete intersection in $K\left[x_{1}, \ldots, x_{m}\right]$, then

$$
\begin{aligned}
H_{I^{n}}(t)= & \sum_{j=1}^{n}\left[\left(\begin{array}{c}
t-\alpha(n-j)-\beta j+(m-1) \\
(m-1)
\end{array}\right)\right. \\
& \left.-\left(\begin{array}{c}
t-\alpha j-\beta(n+1-j)+(m-1) \\
(m-1)
\end{array}\right)\right]+\left(\begin{array}{c}
t-n \alpha+(m-1) \\
(m-1)
\end{array}\right) .
\end{aligned}
$$

Setting $l:=\beta-\alpha$,

$$
\begin{aligned}
H_{I^{n}}(t)= & \sum_{j=1}^{n}\left[\left(\begin{array}{c}
t-\alpha n-j l+(m-1) \\
(m-1)
\end{array}\right)\right. \\
& \left.-\left(\begin{array}{c}
t-\alpha(n+1)-l j+(m-1) \\
(m-1)
\end{array}\right)\right]+\left(\begin{array}{c}
t-n \alpha+(m-1) \\
(m-1)
\end{array}\right) .
\end{aligned}
$$


Proposition 3.9. Suppose that we have an ideal $J$ of the form

$$
J=\left(x^{k}, x^{k-1} y^{\lambda_{k-1}}, \ldots, x y^{\lambda_{1}}, y^{\lambda_{0}}\right)
$$

where $\lambda_{0} \geq \lambda_{1} \geq \cdots \geq \lambda_{k-1}$. Then

$$
H_{J}(t)=\sum_{i=0}^{k-1}\left(\begin{array}{c}
t-\lambda_{i}-i+(m-2) \\
(m-2)
\end{array}\right)+\left(\begin{array}{c}
t-k+(m-1) \\
(m-1)
\end{array}\right) .
$$

4. Algorithms and the proposed invariants. In the previous section, we determined the general structure of gin $\left(I^{n}\right)$ where $I$ is a 2 -complete intersection of type $(\alpha, \beta)$ and showed that it was defined by a strictly decreasing sequence of invariants $\left\{\lambda_{i}\right\}$. We also found expressions for $\lambda_{0}$ and $\lambda_{k-1}$ in terms of $n, \alpha$ and $\beta$ (see Theorem 3.1). In this section, we propose algorithms to determine the remaining invariants, and thus the minimal generators of $\operatorname{gin}\left(I^{n}\right)$. Throughout, $l:=\beta-\alpha$.

Each of the algorithms referred to in Theorem 1.1, and thus the invariants $\lambda_{i}$ that they produce and the resulting gaps $g_{i}:=\lambda_{i-1}-\lambda_{i}$, can be divided into three consecutive phases which we refer to as the Build, the Pattern and the Reverse Build. As the names of the phases suggest, the gap sequences $\left\{g_{i}\right\}$ arising from the Reverse Build and the Build are almost mirror images of each other, while the gap sequences arising from the Pattern consist of a number of repeats of the same sub-sequence called a Pattern Block.

4.1. Algorithms producing the proposed invariants. Given three positive integers $n, \alpha$ and $\beta$ where $n \geq 2$ and $\beta \geq \alpha$, the following algorithms produce a sequence of positive integers $\lambda_{0}, \ldots, \lambda_{k-1}$ which Theorem 1.1 claims are the invariants of $\operatorname{gin}\left(I^{n}\right)$ for a type $(\alpha, \beta)$ complete intersection $I$. For examples of the outputs of these algorithms see Table 4.1. The subroutines called by the algorithms appear after Algorithm 6.

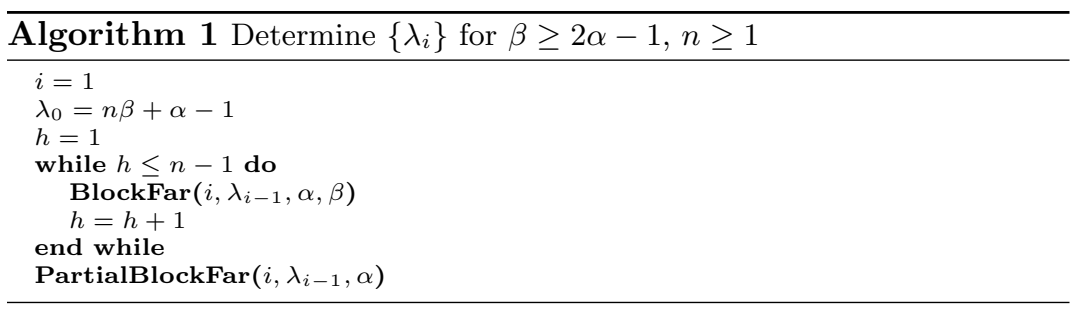



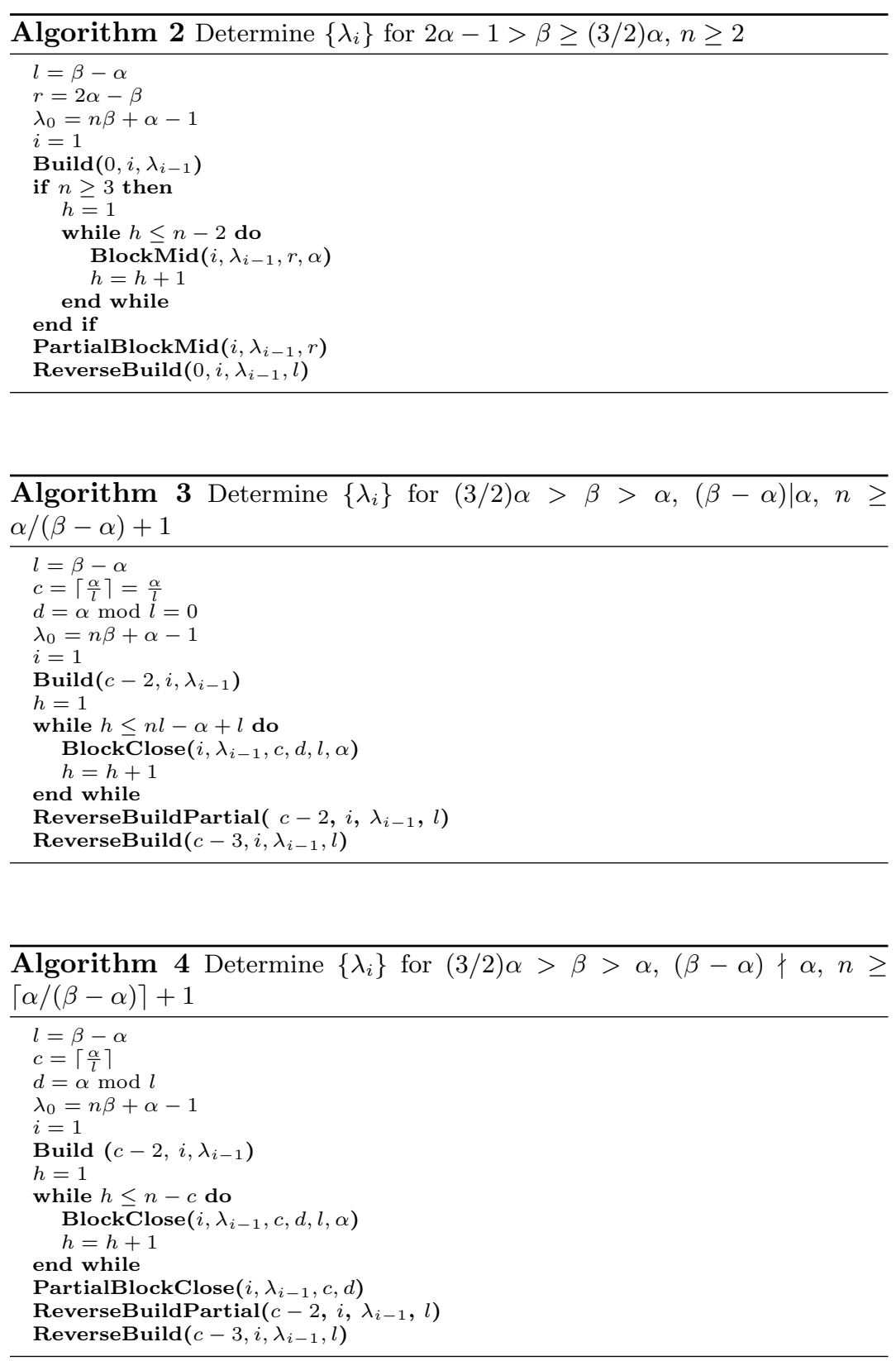

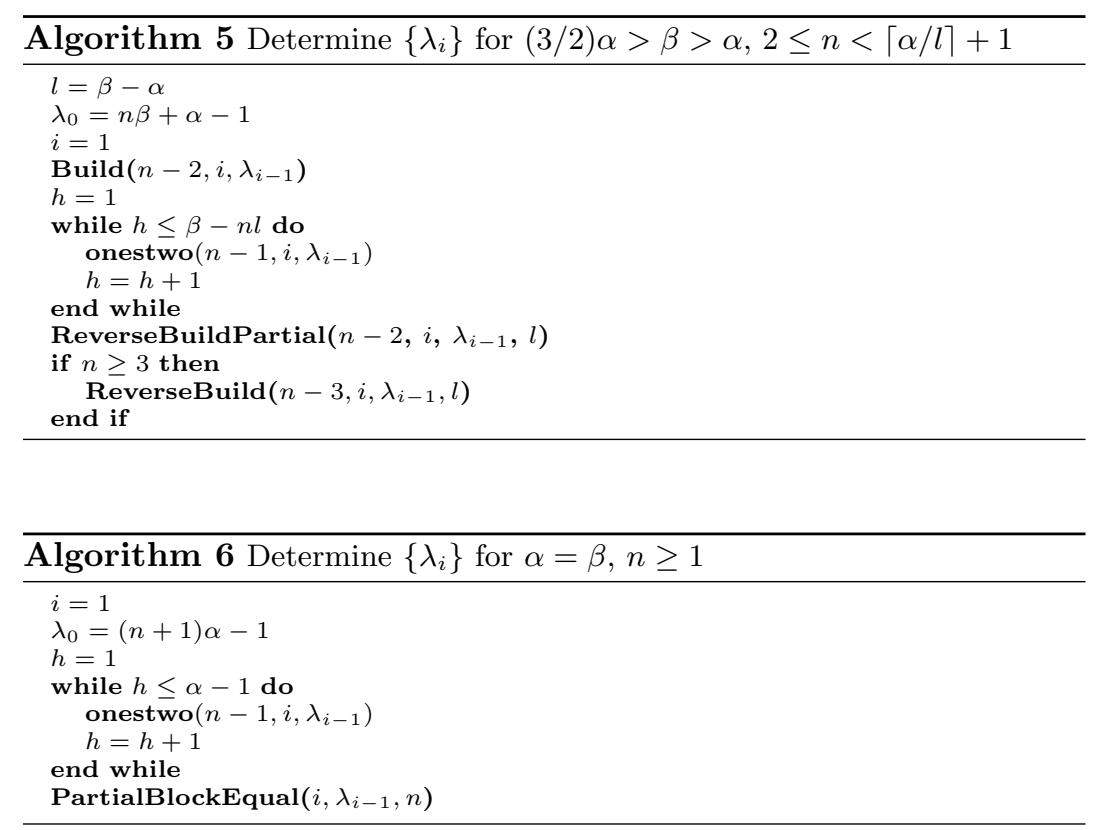

Sub-routines (in alphabetical order)

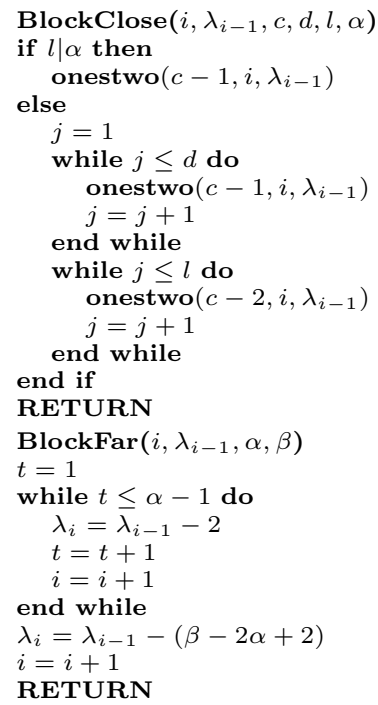




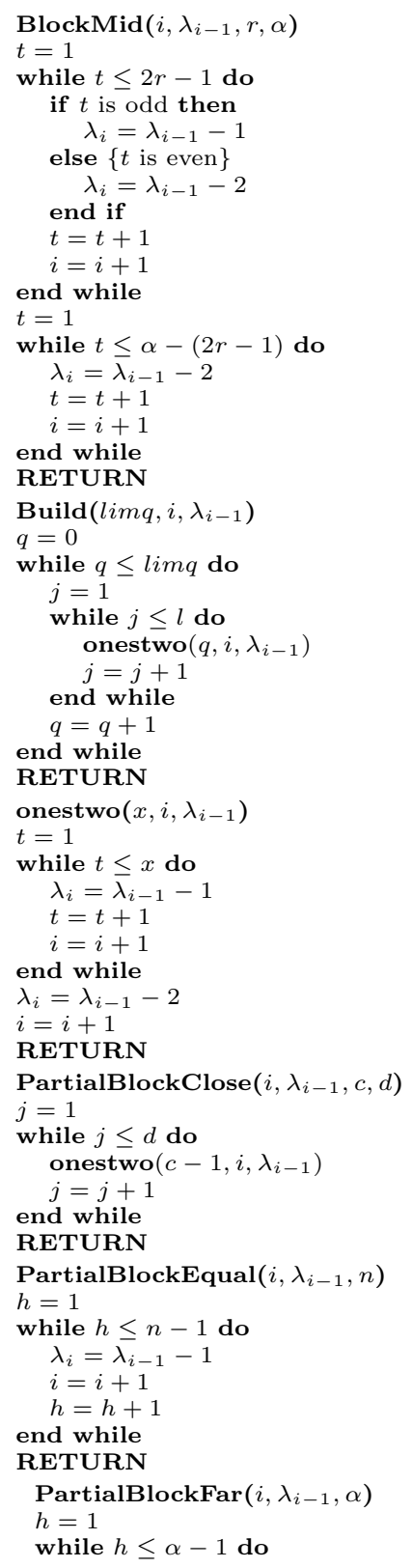




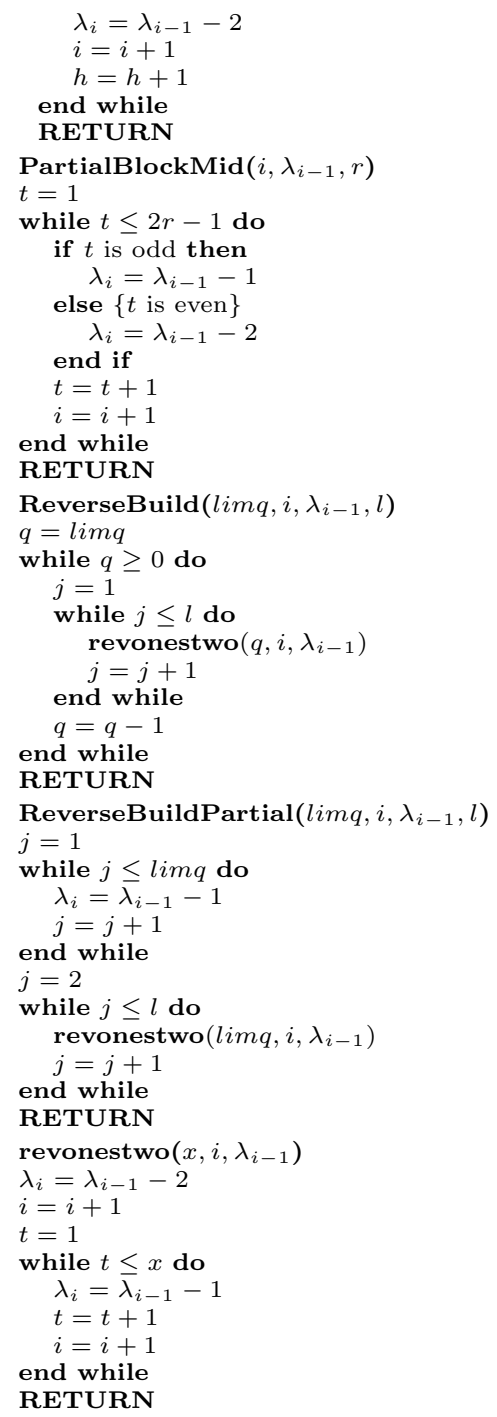

4.2. Description of the algorithms. We will call the $\lambda_{i}$ s produced by these algorithms the proposed invariants of gin $\left(I^{n}\right)$. Each of the algorithms can be divided into the following three stages: 
(1) the Build (absent in the cases where $\beta \geq 2 \alpha-1$ and $\alpha=\beta$ );

(2) the Pattern (consists of full or partial repetitions of a Pattern Block $\left.^{1}\right)$; and

(3) the Reverse Build (also absent in the cases where $\beta \geq 2 \alpha-1$ and $\alpha=\beta)$.

It will be convenient to divide the proposed invariants produced by an algorithm into the same three categories; for example, a $\lambda_{i}$ produced by the Build stage of an algorithm will be said to be part of the Build.

Each of the algorithms begins with defining $\lambda_{0}=n \beta+\alpha-1$ (see Theorem 3.1). The other invariants are obtained one-by-one in the subroutines by subtracting 1,2 or $\beta-2 \alpha+2$ from the previous invariant in the sequence. Patterns in the sequences $\left\{\lambda_{0}, \ldots, \lambda_{k-1}\right\}$ emerge by looking at the sequences of gaps between the $\lambda_{i}$ s; thus, we set $g_{i}$ to be equal to the number subtracted from $\lambda_{i-1}$ to obtain $\lambda_{i}$ or $g_{i}:=\lambda_{i-1}-\lambda_{i}$. The sequence $\left\{g_{i}\right\}$ will be called the gap sequence corresponding to the sequence of proposed invariants. Note that this sequence will consist entirely of the numbers 1,2 and $\beta-2 \alpha+2$; a gap of $\beta-2 \alpha+2$ occurs only when the BlockFar subroutine is called.

Observe the following:

- Since all of the numbers $g_{i}$ are greater than 0 , the sequences $\left\{\lambda_{i}\right\}$ produced by the algorithms are strictly decreasing.

- The gap sequence of the Build written backwards generally gives the gap sequence of the Reverse Build. The exception to this is in the algorithms corresponding to the cases where $(3 / 2) \alpha>\beta>\alpha$. In these cases, everything but the final gap of the Build is reflected in the Reverse Build as seen in the ReverseBuildPartial subroutine.

- The gap sequences of the Build, the Reverse Build, and the Pattern Blocks, are independent of $n$ except in Algorithms 5 and 6 . The only part of the other algorithms that changes as $n$ increases is the number of times that the Pattern Block is repeated.

- The last $\lambda_{i}$ produced by the algorithms is $\lambda_{k-1}=\beta-\alpha+1$. Note that, by Theorem 3.1, this condition must be satisfied for the algorithms to produce the invariants of $\operatorname{gin}\left(I^{n}\right)$. We can check that this condition holds by showing that $\sum_{i=1}^{k-1} g_{i}=$ $\lambda_{0}-\lambda_{k-1}=(n-1) \beta+2 \alpha-2$. 
- The conditions on $\alpha$ and $\beta$ ensure that the algorithms make sense. For example, in Algorithm 3 ReverseBuild $(c-3, \ldots)$ is well defined because, when $(3 / 2) \alpha>\beta>\alpha, c=(\alpha / l)>$ $\alpha /(3 \alpha / 2-\alpha)=2$.

4.3. Asymptotic behavior of the generic initial system. The asymptotic behavior of a graded system of monomial ideals may be described by a geometric object called its limiting shape. If $P_{J} \subseteq \mathbb{R}_{\geq 0}^{n}$ denotes the Newton polytope of a monomial ideal $J$, the limiting shape $P$ of a graded system of monomial ideals $\mathrm{a}_{\bullet}$ is the limit of the scaled polytopes $(1 / q) P_{\mathrm{a}_{q}}: P=\overline{\bigcup_{q \in \mathbb{N} *}(1 / q) P_{\mathrm{a}_{q}}}$.

One corollary of Theorem 1.1 is that the limiting shape of $\left\{\operatorname{gin}\left(I^{n}\right)\right\}_{n}$ when $I$ is a complete intersection of type $(\alpha, \beta)$ is the region of $\mathbb{R}^{2}$ lying above the line passing through the points $(\alpha, 0)$ and $(0, \beta)$. More generally, if $I$ is a complete intersection of type $\left(\alpha_{1}, \alpha_{2}, \ldots, \alpha_{m}\right)$, the limiting shape of the generic initial system of $I$ is the region of $\mathbb{R}^{m}$ lying above the hyperplane passing through the points $\left(\alpha_{1}, 0, \ldots, 0\right), \ldots(0,0, \ldots$, $\left.0, \alpha_{m}\right)([\mathbf{1 3}])$.

5. Proof of Theorem 1.1. In this section we will sketch the proof of Theorem 1.1. The proof in each case referred to in the theorem will involve the following steps:

(1) write non-recursive formulas for the proposed $\lambda_{i}$ from the algorithm;

(2) compute $H_{J}(t)$ where $J=\left(x^{k}, x^{k-1} y^{\lambda_{k-1}}, \ldots, x y^{\lambda_{1}}, y^{\lambda_{0}}\right)$ and the invariants $\lambda_{i}$ are as above; and

(3) rewrite $H_{I^{n}}(t)$ in an appropriate form, sometimes using the assumptions on $\alpha$ and $\beta$, and simplify the expression to show that it is equal to $H_{J}(t)$. By Lemma 3.6, this will prove that $J=\operatorname{gin}\left(I^{n}\right)$ so that the invariants produced by the algorithm are the invariants of $\operatorname{gin}\left(I^{n}\right)$.

Since the required calculations are routine, long and similar in each case, we only include the proof for the case where $2 \alpha-1>\beta \geq(3 / 2) \alpha$, corresponding to Algorithm 2. For convenience, we will divide the formulas and long calculations into parts according to whether they involve invariants and indexing from the Build, Pattern, or Reverse Build as described in subsection 4.2. As before, $l=\beta-\alpha$ and $g_{i}=\lambda_{i-1}-\lambda_{i}$. 


\begin{tabular}{|c|c|c|c|c|c|c|}
\hline 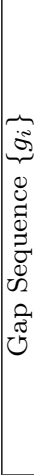 & 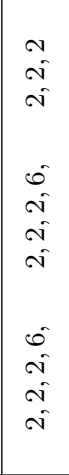 & 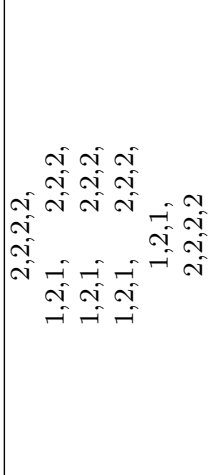 & 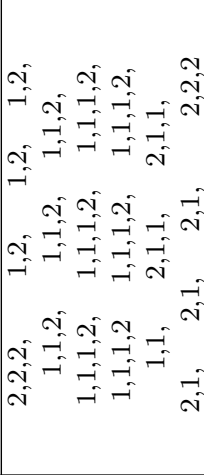 & 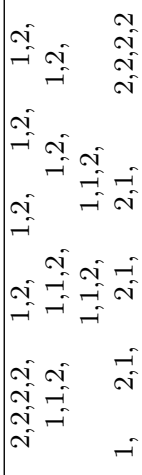 & 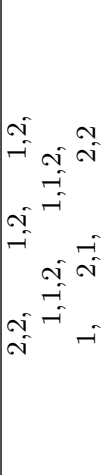 & $=$ \\
\hline 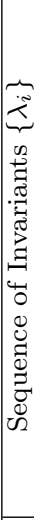 & 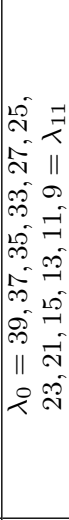 & 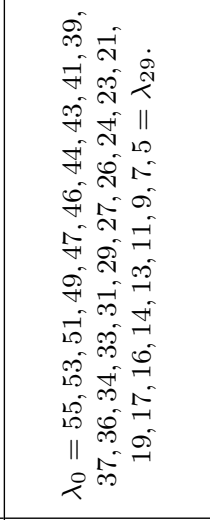 & 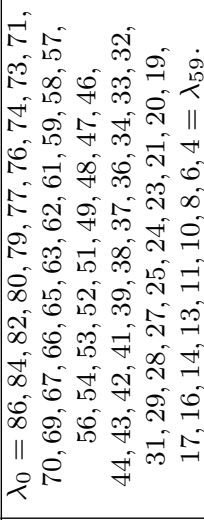 & 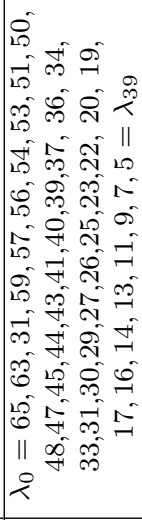 & 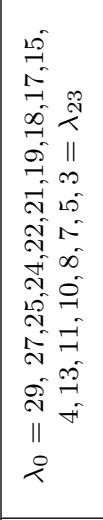 & 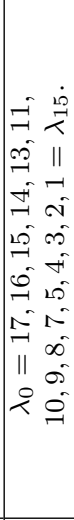 \\
\hline$\approx$ & $\infty$ & 10 & 20 & $\vec{r}$ & $\infty$ & 10 \\
\hline $\begin{array}{l}\text { कृ } \\
\text { ச }\end{array}$ & $\begin{array}{l}\text { I } \\
\text { İ }\end{array}$ & $\begin{array}{l}\hat{0} \\
\stackrel{1}{0} \\
0\end{array}$ & $\begin{array}{l}\stackrel{12}{2} \\
\stackrel{-1}{\rightleftharpoons}\end{array}$ & $\begin{array}{l}\stackrel{\Im}{\leftrightarrows} \\
\stackrel{-}{\Xi}\end{array}$ & 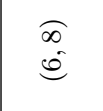 & $\begin{array}{l}\text { ⿵人} \\
\hat{x}\end{array}$ \\
\hline 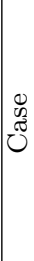 & 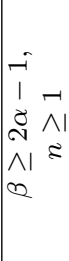 & 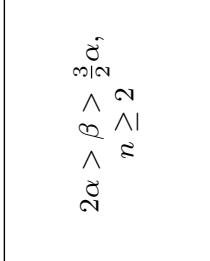 & 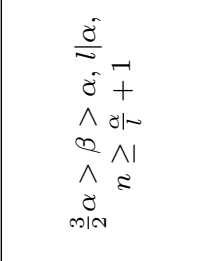 & 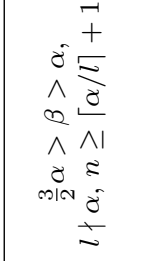 & 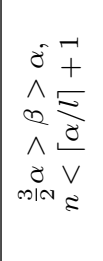 & $\begin{array}{l}\overrightarrow{1} \\
\hat{\imath} \\
\varepsilon \\
\infty \\
\| \\
0\end{array}$ \\
\hline 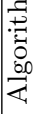 & -1 & $N$ & $\infty$ & $\forall$ & 10 & 0 \\
\hline
\end{tabular}


5.1. Algorithm 2: The case $2 \alpha-1>\beta \geq(3 / 2) \alpha, n \geq 2$. Throughout this section, we will set $r:=2 \alpha-\beta>0$.

5.1.1. Formulas for the proposed invariants. First we will write closedform expressions for the numbers $\lambda_{i}$ produced by Algorithm 2. We distinguish the formulas for invariants produced by the Build, the Reverse Build, and the Pattern phases of Algorithm 2.

Formula for $\lambda_{i}$ in the Build. For $v=0, \ldots, l, \lambda_{v}=\alpha(n+1)+l \cdot n-1-2 v$.

Formulas for $\lambda_{i}$ in the Reverse Build. For $v=k-i=n \alpha-i$ where $i=1, \ldots, l+1, \lambda_{v}=l+1+2(i-1)=l+2 i-1$. Note that $\lambda_{k-i}+k-i=l+n \alpha+i-1$.

Formulas for $\lambda_{i}$ in the Pattern.

(1) For $v=l+j \alpha+y$ where $j=0, \ldots,(n-3)$ and $y=2 r-1, \ldots, \alpha-1$, $\lambda_{v}=\lambda_{0}-[2 l+(2 \alpha-r) j+2 y-r]=\lambda_{0}-[2 l+(\alpha+l) j+2 y-(\alpha-l)]$.

2 For $v=l+j \alpha$ where $j=1, \ldots, n-2$,

$$
\lambda_{v}=\lambda_{0}-\left[j(2 \alpha-r)+2 l=\lambda_{0}-[j(l+\alpha)+2 l]\right] .^{2}
$$

(3) For $v=l+j \alpha+2 p$ where $j=0, \ldots, n-2$ and $p=1, \ldots, r-1=$ $\alpha-l-1$,

$$
\lambda_{v}=\lambda_{0}-[2 l+(\alpha+l) j+2 p+p] .
$$

$(4$ For $v=l+j \alpha+2 p-1$ where $j=0, \ldots,(n-2)$ and $p=1, \ldots, r-1$,

$$
\lambda_{v}=\lambda_{0}-[2 l+(\alpha+l) j+2 p-2+p] .
$$

5.1.2. The Hilbert function of $J$. Suppose that $J=\left(x^{k}, x^{k-1} y^{\lambda_{k-1}}\right.$, $\left.\ldots, x y^{\lambda_{1}}, y^{\lambda_{0}}\right)$ where the $\lambda_{i}$ are the invariants produced by Algorithm 2 and are given by the formulas in subsection 5.1.1. By Proposition 3.9,

$$
H_{J}(t)=\sum_{i=0}^{k-1}\left(\begin{array}{c}
t-\lambda_{i}-i+(m-2) \\
(m-2)
\end{array}\right)+\left(\begin{array}{c}
t-k+(m-1) \\
(m-1)
\end{array}\right) .
$$

Set $X_{j}:=t-n \alpha-j l$ and $Y_{j}:=t-(n+1) \alpha-j l$. Substituting the formulas from subsection 5.1.1, when $n \geq 3$,

$$
H_{J}(t)=\sum_{v=0}^{l}\left(\begin{array}{c}
t-[(n+1) \alpha+n l-1-v]+m-2 \\
m-2
\end{array}\right)
$$




$$
\begin{aligned}
& +\sum_{i=1}^{l+1}\left(\begin{array}{c}
t-[l+n \alpha+i-1]+m-2 \\
m-2
\end{array}\right) \\
& +\sum_{j=0}^{n-3} \sum_{y=2 r-1}^{\alpha-1}\left(\begin{array}{c}
t-[(n+2) \alpha+l(n-j-2)-1-y]+m-2 \\
m-2
\end{array}\right) \\
& +\sum_{j=1}^{n-2}\left(\begin{array}{c}
t-[(n+1) \alpha+l(n-j-1)-1]+m-2 \\
m-2
\end{array}\right) \\
& +\sum_{j=0}^{n-2} \sum_{p=1}^{\alpha-l-1}\left(\begin{array}{c}
t-[(n+1) \alpha+(n-j-1) l-1-p]+m-2 \\
m-2
\end{array}\right) \\
& +\sum_{j=0}^{n-2} \sum_{p=1}^{\alpha-l-1}\left(\begin{array}{c}
t-[(n+1) \alpha+l(n-j-1)-p]+m-2 \\
m-2
\end{array}\right) \\
& +\left(\begin{array}{c}
t-n \alpha+m-1 \\
m-1
\end{array}\right) \\
& =\sum_{j=1}^{n}\left(\begin{array}{c}
X_{j}+m-1 \\
m-1
\end{array}\right)-\sum_{j=1}^{n}\left(\begin{array}{c}
Y_{j}+m-1 \\
m-1
\end{array}\right)+\left(\begin{array}{c}
t-n \alpha+m-1 \\
m-1
\end{array}\right) .
\end{aligned}
$$

When $n=2$,

$$
\begin{aligned}
H_{J}(t)= & \left(\begin{array}{c}
X_{2}+m-1 \\
m-1
\end{array}\right)-\left(\begin{array}{c}
Y_{1}+m \\
m-1
\end{array}\right)+\left(\begin{array}{c}
X_{2}+m-2 \\
m-1
\end{array}\right) \\
& -\left(\begin{array}{c}
Y_{1}+m-1 \\
m-1
\end{array}\right)+\left(\begin{array}{c}
Y_{n-1}+m \\
m-1
\end{array}\right)-\left(\begin{array}{c}
Y_{n}+m-1 \\
m-1
\end{array}\right) \\
& +\left(\begin{array}{c}
X_{1}+m-1 \\
m-1
\end{array}\right)-\left(\begin{array}{c}
X_{2}+m-2 \\
m-1
\end{array}\right)+\left(\begin{array}{c}
t-2 \alpha+m-1 \\
m-1
\end{array}\right) \\
= & \left(\begin{array}{c}
X_{2}+m-1 \\
m-1
\end{array}\right)-\left(\begin{array}{c}
Y_{1}+m-1 \\
m-1
\end{array}\right)-\left(\begin{array}{c}
Y_{2}+m-1 \\
m-1
\end{array}\right) \\
& +\left(\begin{array}{c}
X_{1}+m-1 \\
m-1
\end{array}\right)+\left(\begin{array}{c}
t-2 \alpha+m-1 \\
m-1
\end{array}\right) .
\end{aligned}
$$

5.1.3. The Hilbert function of $I^{n}$. By Proposition 3.8,

$$
H_{I^{n}}(t)=\sum_{j=1}^{n}\left(\left(\begin{array}{c}
t-\alpha n-j l+(m-1) \\
(m-1)
\end{array}\right)\right.
$$




$$
\left.-\left(\begin{array}{c}
t-\alpha(n+1)-l j+(m-1) \\
(m-1)
\end{array}\right)\right)+\left(\begin{array}{c}
t-n \alpha+m-1 \\
m-1
\end{array}\right)
$$

Thus, $H_{I^{n}}(t)=H_{J}(t)$ so, by Lemma $3.6, J=\operatorname{gin}\left(I^{n}\right)$. Therefore, Algorithm 2 produces the invariants of $\operatorname{gin}\left(I^{n}\right)$ when $\beta \geq 2 \alpha-1$ and $n \geq 1$.

\section{ENDNOTES}

1. As their names suggest, BlockFar, BlockMid, and BlockClose are Pattern Blocks. In the cases where $\alpha=\beta$ and $(3 / 2)>\beta>\alpha$, $2 \leq n<\lceil\alpha / l\rceil+1$, the Pattern Block is simply the onestwo subroutine.

2. Note that when $n=2$, the ranges for $j$ in $\mathbf{0}$ and $(2$ are empty. This reflects the fact that Algorithm 2 only includes the Partial Pattern Block when $n=2$.

\section{REFERENCES}

1. J. Ahn, Y.H. Cho and J.P. Park, Generic initial ideals of artinian ideals having Lefschetz properties or the strong Stanley property, J. Algebra 318 (2007), 589-606.

2. J. Ahn and J.C. Migliore, Some geometric results arising from the Borel fixed property, J. Pure Appl. Algebra 209 (2007), 337-360.

3. D. Bayer and M. Stillman, A criterion for detecting m-regularity, Invent. Math. 87 (1987), 1-11.

4. A theorem on refining division orders by the reverse lexicographic order, Duke J. Math. 55 (1987), 321-328.

5. Y.H. Cho and J.P. Park, Conditions for generic initial ideals to be almost reverse lexicographic, J. Algebra 319 (2008), 2761-2771.

6. M. Cimpoeaş, Generic initial ideal for complete intersections of embedding dimension three with strong Lefschetz property, 2006, arXiv:math/0610649v1 [math.AC].

7. A. Conca and T. Romer, Generic initial ideals and fibre products, Osaka J. Math 47 (2010), 17-32.

8. S. Eliahou and M. Kervaire, Minimal resolutions of some monomial ideals, J. Algebra 129 (1990), 1-25.

9. A. Galligo, A propos du théorem de préparation de Weierstrass, Lect. Notes Math. 409 (1974), 543-579.

10. M. Green, Generic initial ideals, in Six lectures on commutative algebra, J. Elias, et al., eds., Springer, New York, 1998.

11. E. Guardo and A. Van Tuyl, Powers of complete intersections: graded Betti numbers and applications, Illinois J. Math. 49 (2005), 265-279. 
12. J. Herzog and H. Srinivasan, Bounds for multiplicities, Trans. Amer. Math. Soc. 350 (1998), 2879-2902.

13. S. Mayes, The limiting shape of the generic initial system of a complete intersection, J. Commutative Algebra, to appear.

Quest University Canada, 3200 University Blvd, Squamish, BC V8B 0N8 Ontario, Canada

Email address: sarah.mayes-tang@questu.ca 